\title{
GCU
}

Glasgow Caledonian

University

University for the Common Good

\section{Time-resolved fluorescence microscopy to study biologically related applications using sol-gel derived and cellular media}

Toury, Marion; Chandler, Lin; Allison, Archie; Campbell, David; McLoskey, David; HolmesSmith, A. Sheila; Hungerford, Graham

Published in:

Proceedings of SPIE Volume 7903: Multiphoton Microscopy in the Biomedical Sciences XI

DOI:

$10.1117 / 12.882088$

Publication date:

2011

Document Version

Publisher's PDF, also known as Version of record

Link to publication in ResearchOnline

Citation for published version (Harvard):

Toury, M, Chandler, L, Allison, A, Campbell, D, McLoskey, D, Holmes-Smith, AS \& Hungerford, G 2011, Timeresolved fluorescence microscopy to study biologically related applications using sol-gel derived and cellular media. in A Periasamy, K König \& PTC So (eds), Proceedings of SPIE Volume 7903: Multiphoton Microscopy in the Biomedical Sciences XI. SPIE, SPIE Multiphoton Microscopy in the Biomedical Sciences XI Conference, San Francisco, United States, 22/01/11. https://doi.org/10.1117/12.882088

\section{General rights}

Copyright and moral rights for the publications made accessible in the public portal are retained by the authors and/or other copyright owners and it is a condition of accessing publications that users recognise and abide by the legal requirements associated with these rights.

Take down policy

If you believe that this document breaches copyright please view our takedown policy at https://edshare.gcu.ac.uk/id/eprint/5179 for details of how to contact us. 


\title{
Time-resolved fluorescence microscopy to study biological related applications using sol-gel derived and cellular media
}

\author{
Marion Toury ${ }^{\mathrm{a}}$, Lin Chandler ${ }^{\mathrm{b}}$, Archie Allison ${ }^{\mathrm{a}}$, David Campbell ${ }^{\mathrm{a}}$, David McLoskey ${ }^{\mathrm{a}}$, \\ A. Sheila Holmes-Smith ${ }^{c}$ and Graham Hungerford ${ }^{a^{*}}$ \\ ${ }^{a}$ HORIBA Jobin Yvon IBH Ltd, 45 Finnieston Street, Glasgow G3 8JU, UK \\ ${ }^{\mathrm{b}}$ HORIBA Scientific Inc, Edison, New Jersey, USA \\ ${ }^{c}$ Dept Biological and Biomedical Sciences, Glasgow Caledonian University, Cowcaddens Road, \\ Glasgow G4 0BA, UK
}

\begin{abstract}
Fluorescence microscopy provides a non-invasive means for visualising dynamic protein interactions. As well as allowing the calculation of kinetic processes via the use of time-resolved fluorescence, localisation of the protein within cells or model systems can be monitored. These fluorescence lifetime images (FLIM) have become the preferred technique for elucidating protein dynamics due to the fact that the fluorescence lifetime is an absolute measure, in the main independent of fluorophore concentration and intensity fluctuations caused by factors such as photobleaching. In this work we demonstrate the use of a time-resolved fluorescence microscopy, employing a high repetition rate laser excitation source applied to study the influence of a metal surface on fluorescence tagged protein and to elucidate viscosity using the fluorescence lifetime probe DASPMI. These were studied in a cellular environment (yeast) and in a model system based on a sol-gel derived material, in which silver nanostructures were formed in situ using irradiation from a semiconductor laser in $\mathrm{CW}$ mode incorporated on a compact time-resolved fluorescence microscope (HORIBA Scientific DeltaDiode and DynaMyc).
\end{abstract}

Keywords: DASPMI, FLIM, Silver nanostructure, Sol-gel, Surface enhanced fluorescence, Yeast.

\section{INTRODUCTION}

\subsection{Fluorescence lifetime imaging}

The use of the fluorescence lifetime as a means to provide contrast in microscopy has increased markedly in recent times $^{1}$. Its usage adds the sensitivity of fluorescence and the fact that the measurement of a fluorescence lifetime provides an absolute value to this area of microscopy. This is advantageous as it is largely independent of fluorophore concentration and can offset the effect of photobleaching. Many applications of fluorescence have been in the field of biological or life sciences. Principally, as fluorescence is a minimally invasive technique, allowing the characterisation of the local environment present within biological tissues. This can be in terms of the measurement of $\mathrm{pH}$, calcium ion concentration and oxygen, for example, or the elucidation of protein and other molecular interactions. A major method for studying the latter is the use of Förster resonance energy transfer (FRET). These measurements can be employed to add contrast between different regions of interest or to make use of the decay data to determine specific parameter values. FLIM is not only applied to "life sciences" but is also becoming widely applied to different areas of materials science.

The increased use of FLIM is related to the availability of equipment, either in the form of specifically built microscopes or the use of add-on units to convert conventional fluorescence microscopes to measure fluorescence lifetimes. Historically the most sensitive means of fluorescence lifetime determination is the time-correlated single-photon counting (TCSPC) technique. This is based on the detection of arrival times of individual photons after optical excitation, which at low signal levels upon repeated excitation-collections allows a histogram indicative of the decay 
process to be accumulated. However, it suffers from an inherent disadvantage related to the dead time of the counting electronics, such that high collection rates can lead to "pile up",-4 leading to the generally accepted limiting of the collection rate to $2 \%$ that of the excitation rate. A clear limitation to the speed of data acquisition is therefore the repetition rate of the source and the dead time of the electronics. This has to be taken into consideration in the study of certain samples where stability and/or photobleaching are of concern.

\subsection{Surface enhanced fluorescence}

The effect of proximity of a conducting metal surface to a fluorophore has been shown to modify its fluorescence behaviour. This has been likened to the effect seen in Raman spectroscopy (surface enhanced Raman - SERS) in which an enhancement of the Raman signal of many orders of magnitude can occur ${ }^{5}$. The presence of a metal particle has been shown to have positive effects on the fluorescence signal, such as an increase in intensity, photostability and Förster energy transfer distance ${ }^{6}$. This plasmon effect has also been termed surface or metal enhanced fluorescence ${ }^{7}$ and is the genesis of a recent area of research. This has included the study of fluorescent single molecules ${ }^{8}$ and even longer-lived lanthanide luminescence ${ }^{9}$, although a major focus is the study of biologically important molecules. The presence of a conducting metal surface has been postulated to affect the fluorescence via different possible mechanisms. Changes in the fluorescence behaviour may relate to an increase in the intensity of the electric field in the vicinity of the fluorophore caused by absorption of light by the nanostructure producing an increased rate of excitation. Interaction with the plasmon band and the fluorophore may also result in alteration of the radiative $\left(\Gamma_{\mathrm{m}}\right)$ or non radiative $\left(k_{n r}\right)$ decay rate. At short separations $(<5 \mathrm{~nm})$ between the metallic surface and fluorophore, the major effect is quenching by energy transfer, while increasing separation (up to $\sim 20 \mathrm{~nm}$ ) favours an increase in the radiative decay rate ${ }^{10}$. In the presence of a conducting metal surface the fluorescence lifetime $\left(\tau_{m}\right)$ and quantum yield $\left(Q_{m}\right)$ can be written ${ }^{11}$,

$$
\begin{gathered}
\tau_{m}=\frac{1}{\Gamma_{m}+k_{n r}} \\
Q_{m}=\frac{\Gamma_{m}}{\Gamma_{m}+k_{n r}}
\end{gathered}
$$

The change in both the lifetime and quantum yield on introducing a metallic surface can be denoted by $G_{\tau}$ and $G_{Q}$ respectively, such that

$$
\begin{aligned}
G_{\tau} & =\frac{\tau_{m}}{\tau} \\
G_{Q} & =\frac{Q_{m}}{Q}=N_{r} \frac{\tau_{m}}{\tau}
\end{aligned}
$$

where, $\tau$ and Q are the fluorescence lifetime and quantum yield without the metallic surface present and $N_{r}$ is the radiative enhancement factor ${ }^{11}$

Controlling the different aspects, especially position and distance between the surface and fluorophore, are therefore of importance in the application of this phenomenon.

\subsection{Formation of silver nanostructures in biocompatible sol-gel derived media}

Materials produced using the sol-gel technique ${ }^{12,13}$ have been previously used by one of the authors to incorporate bioactive molecules (proteins / enzymes that can exhibit catalytic activity) ${ }^{14-16}$ and the matrix forming reaction can be readily adapted to include the presence of silver ions within silica films. Media can be produced using this technique at room temperature or lower and exhibit robust glass-like properties and are highly porous and have large internal surface areas. There are several reports of their use for biological applications ${ }^{17}$ which, with ease of manufacture, make them good materials for potential biological sensing applications. The fact that media can be formed at relatively low temperatures lends itself to the possible future inclusion of biomolecules. 
There has been work to incorporate silver particles into silica glass produced by the sol-gel technique, but these have involved a form of thermal treatment ${ }^{18-21}$. The fact that light irradiation has been employed to react the silver ions within microheterogeneous media ${ }^{22,23}$ means that this approach can also be promising if applied to sol-gel derived media. Irradiation of aqueous solutions of silver ions (our sol-gel derived media have been previously shown to retain solvents ${ }^{24,25}$ ) can induce the photo-oxidation of water accompanied by the formation of silver atoms $\left(\mathrm{Ag}^{0}\right)$. These may aggregate to produce clusters $\left(\mathrm{Ag}^{0}\right)_{n}$ and colloids $\left(\mathrm{Ag}^{0}\right)^{\mathrm{c}}$ of silver and can be represented by the following scheme ${ }^{26}$.

$$
\begin{aligned}
& \mathrm{Ag}^{+}+\mathrm{H}_{2} \mathrm{O}+\mathrm{h} v \rightarrow \mathrm{Ag}^{0}+\mathrm{H}^{+}+\mathrm{OH} \bullet \\
& \mathrm{nAg}^{0} \rightarrow\left(\mathrm{Ag}^{0}\right)_{\mathrm{n}} \rightarrow\left(\mathrm{Ag}^{0}\right)^{\mathrm{c}}
\end{aligned}
$$

Thus the combination of a biocompatible material with the incorporation of silver nanostructures could provide a useful substrate for the study of protein interactions and we have begun investigating this ${ }^{27}$. Especially, since the positioning and size of the nanostructures can be controlled via the refinement of the laser irradiation this opens the possibility for "lab on a chip" type applications such as protein / cell recognition via FRET sensing for example.

\subsection{Employment of DASPMI}

The fluorescent dye dimethylamino-styrlmethylpyridinium iodine (DASPMI) has found application as a mitochondria $\operatorname{stain}^{28}$ and its photophysics have been well studied ${ }^{29,30}$. This fluorescence lifetime of this dye has been shown to be useful in indicating the viscosity of its environment and in this aspect it has been employed in the characterisation of the manufacture of sol-gel derived media ${ }^{31,32}$. Even in its use to stain mitochondria, changes in its photophysical behaviour have been noted ${ }^{33}$. The fluorescence quantum yield, $\Phi_{f}$, of this type of probe, sometimes referred to as a molecular rotor, is a function of the viscosity $(\eta)$ of the medium it is dissolved in and can be described using the Förster-Hoffmann equation $^{34}$

$$
\Phi_{f}=z \eta^{\alpha}
$$

where $z$ and $\alpha$ are constants. Making use of the quantum yield

$$
\Phi_{f}=\frac{k_{r}}{k_{r}+k_{n r}}
$$

and the fluorescence lifetime $\tau_{f}$

$$
\tau_{f}=\frac{1}{k_{r}+k_{n r}}
$$

where, $k_{r}$ is the radiative rate constant and $k_{n r}$ is the non-radiative rate constant, equation (5) can then be expressed ${ }^{35}$ as

$$
\tau_{f}=\frac{Z}{k_{r}} \eta^{\alpha}
$$

Taking logarithms in equation (8) leads to

$$
\log \tau_{f}=\alpha \log \eta+\log \left(\frac{z}{k_{r}}\right)
$$

This means that a calibration graph can be obtained plotting $\log \tau_{f}$ versus $\log \eta$, (which yields a straight line) ${ }^{32}$. This allows conversion of fluorescence lifetimes into viscosity, which can be advantageous over the alternative method of fluorescence anisotropy, as it is a simpler measurement to perform. 


\section{EXPERIMENTAL DETAILS}

\subsection{TCSPC equipment description and validation}

The time-resolved fluorescence measurements were performed using a HORIBA Scientific DynaMyc compact fluorescence microscope with confocal ability. In relation to the main TCSPC components, the key elements that enable the ultra high count rates (up to $100 \mathrm{MHz}$ ) are the DeltaDiode excitation source and the FluoroHub time interval measurement electronics.

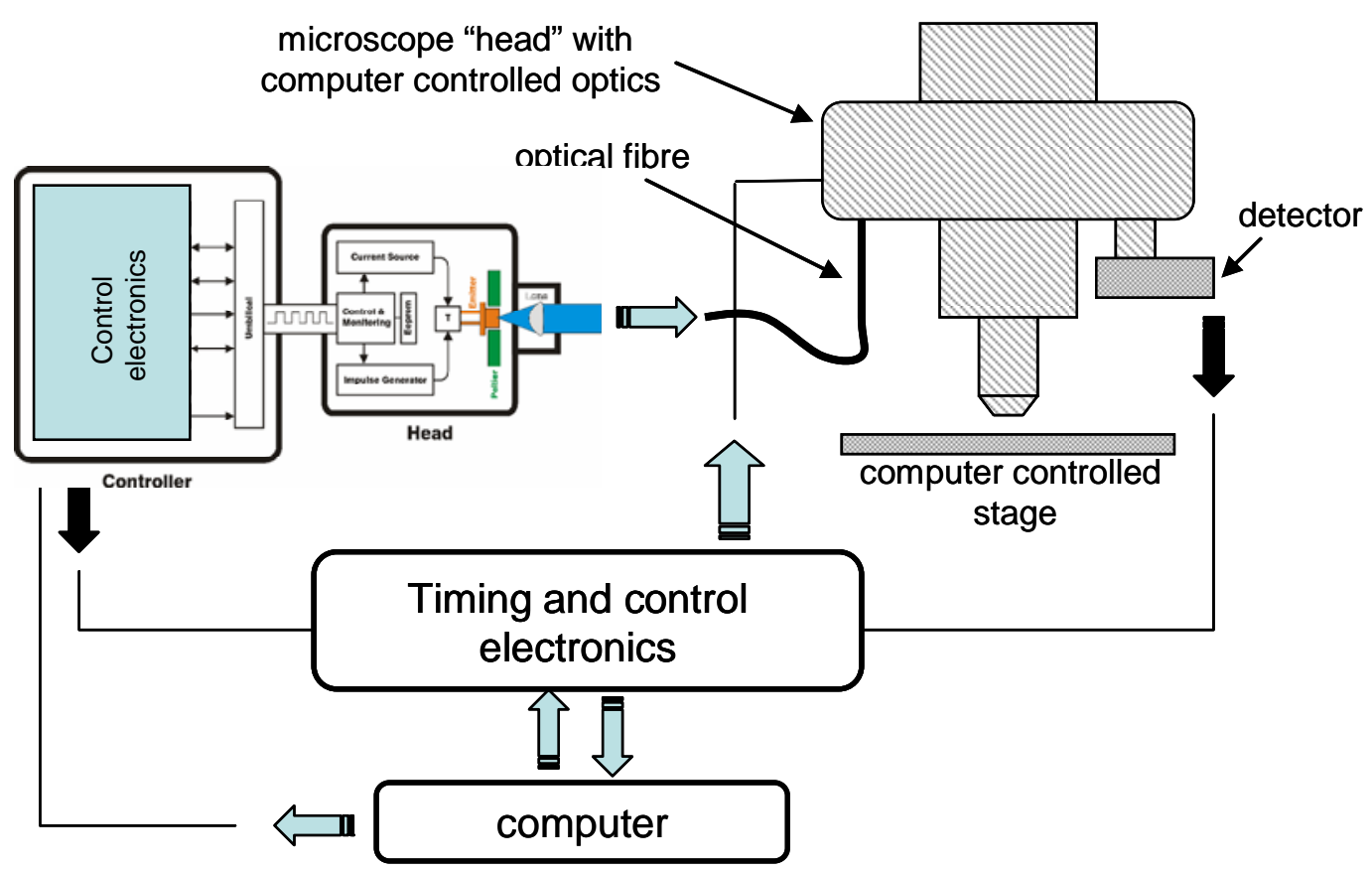

Fig 1. Schematic representation of microscope showing TCSPC components

As shown in Figure 1 an impulse generator produces electrical pulses with 100 ps rise and fall times and, importantly, has a reset time of $<10 \mathrm{~ns}$ allowing pulse repetition rates of $100 \mathrm{MHz}$ to be achieved. Impulses are generated "on command", which means that the instrument can easily be configured to measure a wide range of lifetimes. The FluoroHub contains a time interval measurement (TIM) circuit that determines the arrival time for each detected photon relative to the laser excitation event. The TIM circuit has a dead time of $<10 \mathrm{~ns}$ which is at least one order of magnitude shorter than conventional TCSPC electronics and is perfectly matched to the impulse generator's $<10 \mathrm{~ns}$ reset time. This means that the system can operate towards the upper limit of detector count rate capability ( $>10 \mathrm{Mcps})$, with reduced pile-up losses, addressing one of the historic criticisms of TCSPC. Both sets of electronics contain USB host processors and are entirely software controlled. Factory programming of all calibration data into non volatile memory (eeprom) in the head eliminates the need for manual tuning. The combination of embedded calibration data, hot-swap circuitry and USB interface allow for unprecedented ease of use.

To test the ability of the source and timing electronics, two samples were measured a fluorescence standard, erythosin $\mathrm{B}^{36}$ in water, and a bodipy derivative ${ }^{37}$. These measurements were performed with a HORIBA Scientific TemPro-01 (incorporating a DeltaDiode and FluoroHub) equipped with a MCP detector and the outcome showing the fitted data given in Fig 2. The lifetime values returned from the fit to a single exponential decay model were $85 \mathrm{ps}$ and 375 ps for the erythrosin and bodipy samples respectively, in keeping with those expected. 

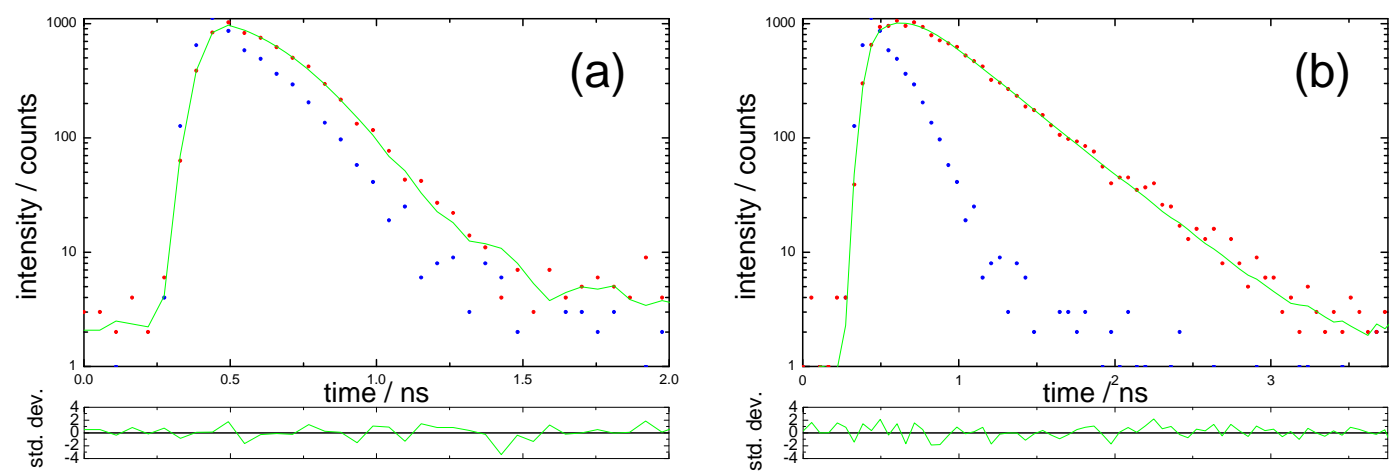

Fig 2. Decay of (a) erythrosin B in water and (b) a bodipy derivative in ethanol. Plots show the fluorescence decay, instrumental response, fitted function (single exponential) and weighted residuals

An important consideration in relation to microscopy is the speed of data acquisition and to show the potential of the source and timing electronics a test to see the shortest acquisition time making use of the $100 \mathrm{MHz}$ excitation rate, using the bodipy derivative as the sample. Prior to this different data collection rates were also tried and it was found that even at a rate $8 \%$ of that of the excitation the influence of pile up as negligible under the conditions used. Previous work has shown that a single exponential decay requires a minimum of 185 counts in order to obtain a lifetime within a $10 \%$ error $^{38}$. Figure 3 shows the outcome for the use of different collection rates and times on the number of photons collected.
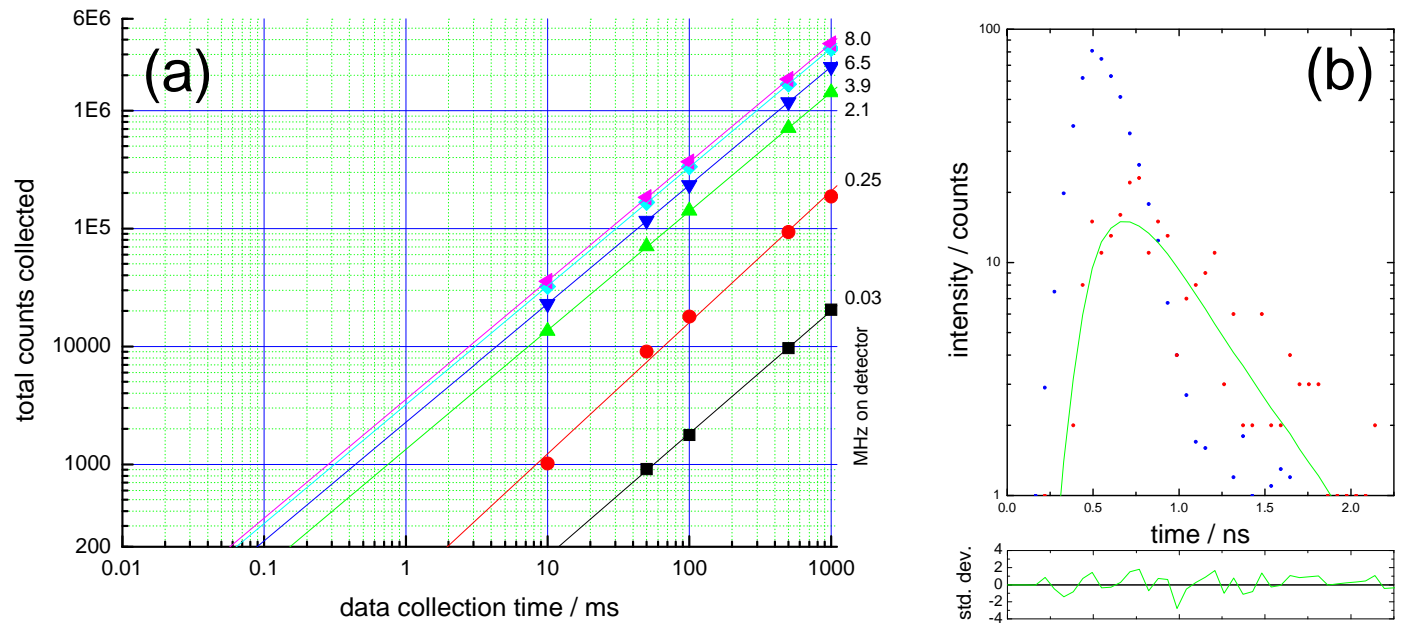

Fig 3. (a) Influence of the data collection time on the number of counts in the measured decay. (b) Decay data for the bodipy derivative measured with $100 \mathrm{MHz}$ excitation frequency with a $7.8 \mathrm{MHz}$ collection rate

Visually it can be seen from this figure that the time to collect sufficient photons to perform an exponential fit analysis is approx $60 \mu \mathrm{s}$. This collection time was used and the data obtained also shown in Figure 3. The lifetime value recovered (336 ps) was within error equivalent to that obtained using more counts and the MCP detector. Thus, this shows the capability of these components to assist in the fast acquisition of time-resolved fluorescence data, although it should be remembered that the actual repetition rate is limited as to not re-excite the sample before it has completely decayed. 


\subsection{Sol-gel derived film preparation}

Silica sol-gel derived films were made using previously described methods ${ }^{16,39}$, with the exception that in the method described in the former method ${ }^{16} 250 \mu \mathrm{l}$ of $2 \mathrm{M} \mathrm{AgNO}_{3}$ was mixed with $2 \mathrm{ml}$ of sol, $2 \mathrm{ml}$ of phosphate buffered saline and $0.5 \mathrm{ml}$ of ethanol and then deposited onto a slide. The latter method ${ }^{39}$ was modified by substituting $2.5 \mathrm{ml}$ of $0.01 \mathrm{M}$ $\mathrm{HNO}_{3}$ (pH 2.2) for the $\mathrm{HCl}$. After mixing the solution was left at $60{ }^{\circ} \mathrm{C}$ for 4 hours before addition of $50 \mu \mathrm{l}$ (unless otherwise stated) of $4 \mathrm{M} \mathrm{AgNO}_{3}$. The films were then produced by dipping. The latter was intended for producing films that could be exposed to UV light $(365 \mathrm{~nm})$ over a large area of the film to facilitate a full spectroscopic characterisation. The former, a method suitable for protein entrapment ${ }^{15}$, was in the main used in the microscope pattering experiment, where only small areas were irradiated. Both sets of films exhibited the same behaviour in terms of laser irradiation.

\subsection{Silver nanostructure formation}

Sol-gel derived films containing $\mathrm{AgNO}_{3}$ were placed on the microscope slide and the DynaMyc's DataStation control software used to position the stage to form the required pattern. The DeltaDiode, with emission at $475 \mathrm{~nm}$, was used in $\mathrm{CW}$ mode ( $\sim 5 \mathrm{~mW}$ power) and the time per point was in the order of 200 seconds (depending on sample).

\subsection{Protein labelling}

Bovine serum albumin (BSA) was labelled with a FITC tag and purified using a FluoroTag ${ }^{\mathrm{TM}}$ FITC conjugation kit from Sigma-Aldrich. The protein to tag labelling ratio was less than one label per protein (verified using UV-vis spectroscopy). Films were immersed in a solution (phosphate buffered saline - PBS - at $\mathrm{pH}$ 7.4) containing the labelled BSA for half an hour and excess solution removed.

\subsection{Yeast preparation}

For demonstrative purposes common baking yeast (Saccharomyces cerevisiae) was used and incubated with DASPMI in aqueous solution at $40^{\circ} \mathrm{C}$ for at least 40 minutes before use. As the lifetime of DASPMI in water is short $(\sim 60 \mathrm{ps})^{33}$ no further purification was performed and differentiation left to the analysis of the time-resolved data. As the energy regime has been found to influence mitochondria morphology ${ }^{40}$ both sucrose and ethanol were added to the yeast solution.

\subsection{Measurements}

Optical examination of the samples was performed using a HORIBA Scientific DynaMyc equipped with a Lumenera Infinity 3-1C CCD camera to collect images using white light excitation, with emission selected via a beam splitter or filter cube. Times 10,50 and 60 objectives were employed. The DynaMyc was used, in multipoint and FLIM modes to obtain time-resolved fluorescence images using a DeltaDiode excitation source emitting at $475 \mathrm{~nm}$, in pulsed mode (16.6 $\mathrm{MHz}$ ), with the emission monitored at $520 \mathrm{~nm}$ or $610 \mathrm{~nm}$ using a TBX-04 picosecond detection module. Fluorescence lifetime data were analysed globally by linking common fluorescence lifetimes using DAS6 software and taken to be a sum of exponentials of the form

$$
I(t)=\sum_{i=1}^{n} \alpha_{i} \exp \left(-t / \tau_{i}\right)
$$

with the average lifetime obtained from

$$
\tau_{\text {ave }}=\Sigma \alpha_{i} \tau_{i}
$$

All measurements were performed at ambient temperature and other fluorescence lifetime data were obtained using a HORIBA Scientific TemPro-01 system. Time-resolved emission spectra (TRES) were analysed globally and when required to account for very short lived decays or scattered light the shorter lived component was fixed to $\sim 50 \mathrm{ps}$. The calculated decay associated spectra are shown smoothed for clarity. 


\section{RESULTS AND DISCUSSION}

\subsection{Sol-gel derived system}

\subsubsection{FITC-BSA interaction with silver nanostructure containing sol-gel derived films}

Films were produced containing silver nitrate and exposed to $\mathrm{CW}$ laser irradiation to promote the formation of silver structures. The formation of these structures has been confirmed via the use of AFM and UV-vis spectroscopy ${ }^{27}$ and their presence observed using microscopy - see Figure 4. The corresponding analysed time-resolved fluorescence images, obtained after adsorption of FITC labelled BSA are also shown in this figure.
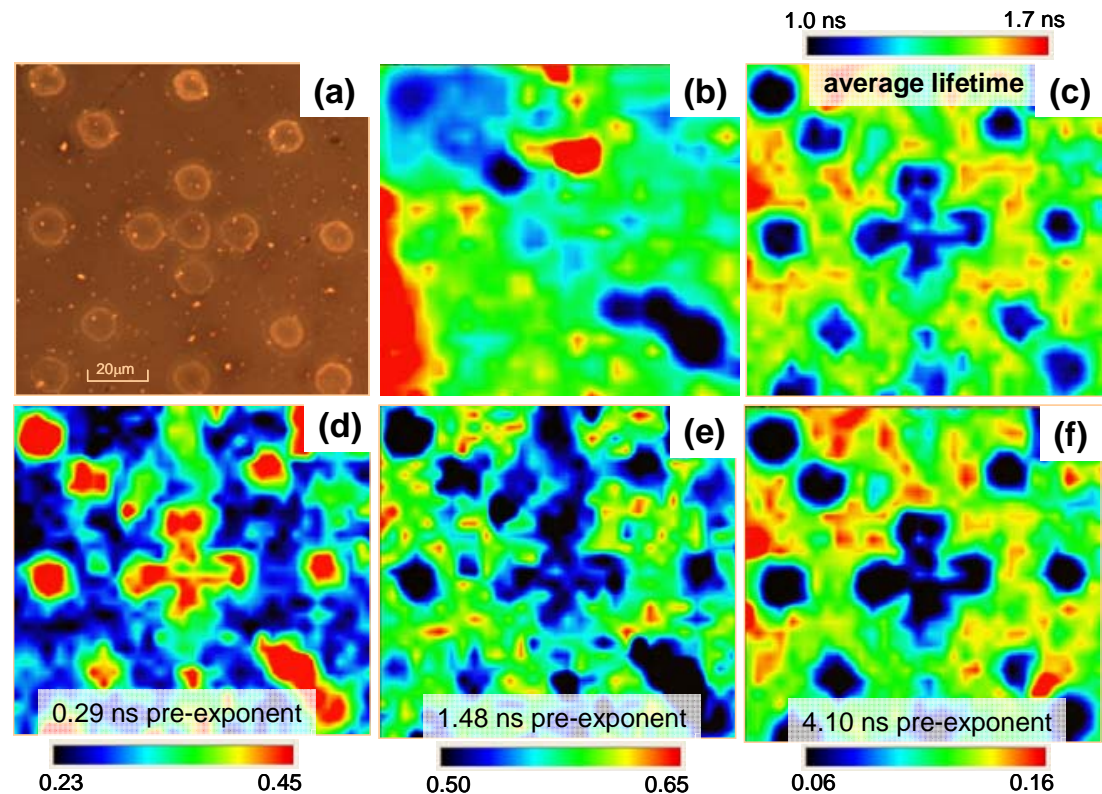

Fig. 4. Bright field image of (a) a sol-gel film after $\mathrm{CW}$ laser irradiation and analysed data from a timeresolved dataset for the emission of FITC labelled to BSA adsorbed to the film surface; (b) FLIM intensity, (c) average lifetime obtained from the 3 exponential analysis using linked lifetimes showing the normalised pre-exponentials for the (d) $0.29 \mathrm{~ns}$, (e) $1.48 \mathrm{~ns}$ and (e) $4.1 \mathrm{~ns}$ lifetime components.

From Figure 4 it can be seen that there is a good correspondence between the position of the silver formations and the fluorescence properties of the FITC label. Overall there is an apparent quenching of the FITC fluorescence in the vicinity of the silver structures. In order to obtain a good fit the data was analysed in terms of three linked lifetimes, whose values were determined by fitting to the sum of all the fluorescence decays. This allowed the determination of the average lifetime, Figure $4 \mathrm{c}$ and the normalised pre-exponential components (Figure 4d,e,f). The two longer lifetime values are similar to those seen for FITC covalently bound to BSA and dominate in the areas without the silver structures. The shorter, quenched, lifetime is predominantly seen in the vicinity of the silver nanostructures.

For this particular dataset, the intensity data is not clearly demonstrative, but it appears that overall the fluorescence intensity is lower at the nanostructure positions (Figure 4b). However we have also reported an observed increase in fluorescence intensity, accompanied by a decrease in the fluorescence lifetime - in keeping with a modest enhancement of the radiative decay rate ${ }^{27}$. The discrepancy of the intensity is easily explained by the fact that we have not yet exercised complete control of the distance between the metal surface and the fluorophore. Most likely the distance in this case is too close, which means that quenching process dominates compared to radiative enhancement which requires a larger separation ${ }^{6,7}$. Overall it is clear that these systems, in which light is used to produce silver structures at specific positions in an easy to produce medium, hold promise in the study of protein interactions in the application field of assays and biosensors. 


\subsection{Cellular system}

\subsubsection{DASPMI doped Saccharomyces cerevisiae}

The investigation of yeast, as well as its obvious application in the food industry, has application in genetic research, where the study and knowledge of its lifecycle ${ }^{41}$ is required. The use of fluorescence microscopy is useful in following this and it has been employed to study the yeast vacuole making use of FITC fluorescence, although this dye has been noted when coupled to a dextran not to be stably retained ${ }^{42}$. As well as being a mitochondria stain that has been employed in yeast ${ }^{40}$, DASPMI also (like the bodipy derivative used earlier in this work) exhibits a lifetime that is indicative of the viscosity of its microenvironment - a factor that some of the authors have previously made use of ${ }^{31,32}$. With this in mind we made use of DASPMI as probes in a demonstrative time-resolved fluorescence microscopy study of Saccharomyces cerevisiae.

A preliminary cuvette time-resolved emission measurement of a yeast sample containing the probe was performed using a HORIBA Scientific TemPro-01, equipped with a DeltaDiode running at a repetition rate of $100 \mathrm{MHz}$. The resultant decays were analysed globally and the decay associated spectra obtained ${ }^{43}$. The outcome is shown in Figure 6.

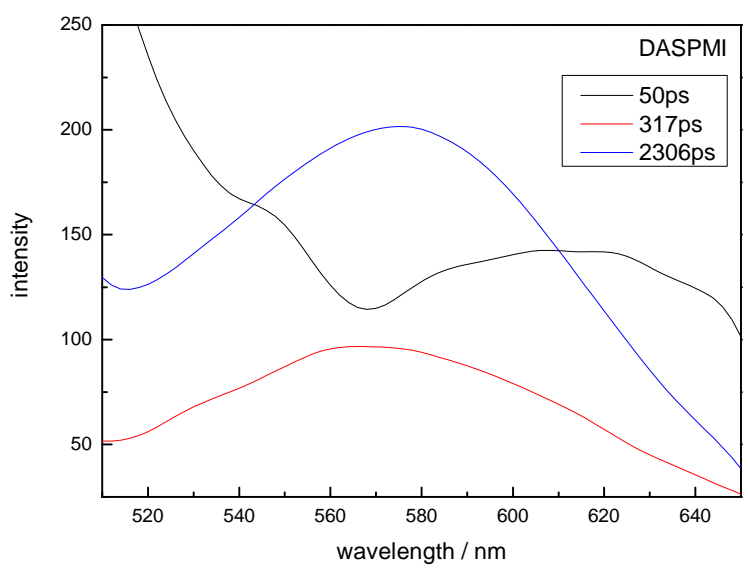

Fig. 6. Decay associated spectra obtained for the DASPMI-yeast system with excitation at $468 \mathrm{~nm}$ using a

DeltaDiode excitation source at a repetition rate of $100 \mathrm{MHz}$.

It should be noted, that from the above figure, the shortest-lived component is influenced by the presence of scattered light. However, it is also possible to easily discern the presence of an emission band. It should be kept in mind that since the sample has not been purified, there is the possibility that some emission may relate to DASPMI in the aqueous solution, but because of its short fluorescence lifetime ${ }^{33}$, this should only be present, if seen, in the spectrum for the shortest-lived component. Indeed, this is probably the origin of the longer wavelength peak seen in this spectrum. The need to use three decay components to fit the data is in keeping with the photophysics of this molecule and relates to the effect of intramolecular charge transfer and bond twisting ${ }^{29,30}$ and the values obtained are similar in magnitude to those found for the dye in endothelial cells ${ }^{33}$. The relatively short wavelength for the peak emission of the longer-lived decay components (plus one close to $545 \mathrm{~nm}$ in the short-lived spectrum) is in keeping with emission from a microenvironment with a low dielectric constant. Making use of the average lifetime as this as previously been employed to calculate viscosity ${ }^{32}$, allowed an estimate of $\sim 23 \mathrm{cP}$ to be made, which is slightly larger than that obtained for the cell interior $(\sim 14$ $\mathrm{cP})^{44}$ and many times higher than that found for a yeast vacuole $(\sim 2.5 \mathrm{cP})^{45}$. However, higher intracellular viscosities have been measured using molecular rotors ${ }^{37}$ and DNA relaxation ${ }^{46}$ with much depending on the location of the probe molecule.

FLIM imaging of the DASPMI-yeast system was made with $475 \mathrm{~nm}$ excitation and monitoring the emission at $610 \mathrm{~nm}$. The subsequent analysis of the time-resolved data, along with that of the camera image, is shown in Figure 7. The complete analysis, made by linking common lifetimes to all the fluorescence decays $(\sim 6300)$, required the sum of three lifetime components to fit the data. A short-lived fluorescence $(\sim 0.16 \mathrm{~ns})$ was returned (data not shown) and found to dominate the region outside the yeast cells and was thus attributed to residual dye in solution. As can be seen from the intensity image (Figure 7) its contribution to the overall fluorescence was negligible, but is seen in the analysis as the measurement of the lifetime is absolute and largely independent of the concentration of a fluorescing species. The other 
fluorescence lifetimes recovered were $\sim 0.42 \mathrm{~ns}$ and $2.5 \mathrm{~ns}$, values in keeping with those obtained via the TRES measurement and showing the presence of a microenvironment much more viscous than water.
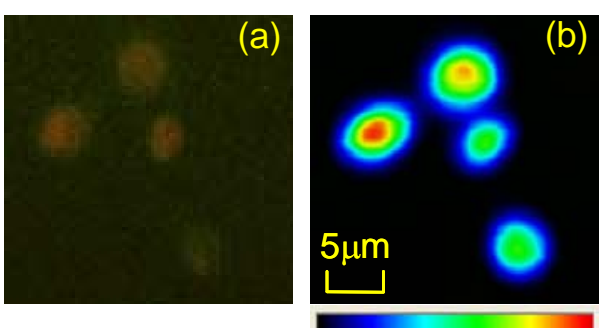

less

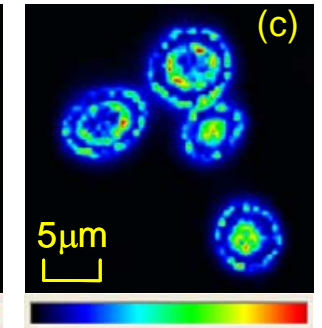

more $0.21 \mathrm{~ns}$

$1.28 \mathrm{~ns} \quad 0.13$
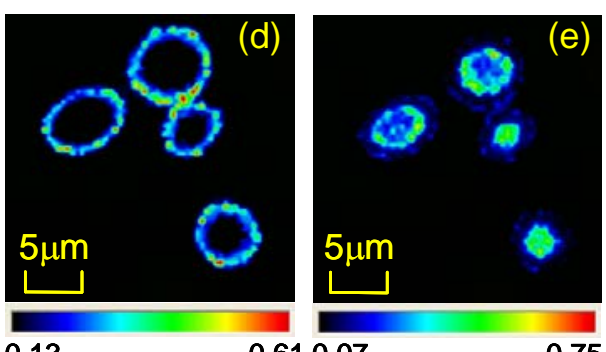

Fig. 7. DASPMI stained yeast excited at $475 \mathrm{~nm}$ and monitored at $610 \mathrm{~nm}$. (a) fluorescence camera image, (b) FLIM intensity image and data recovered from the fitting procedure; (c) average lifetime map,

(d) pre-exponential contribution for a $0.42 \mathrm{~ns}$ lifetime and (e) pre-exponential contribution for a $2.5 \mathrm{~ns}$ lifetime.

Both the camera and FLIM intensity images show the presence of fluorescence within the yeast cell. However looking at the images obtained from the fitting procedure (Figure $7 \mathrm{c}, \mathrm{d}, \mathrm{e}$ ), two different regions can be differentiated in terms of fluorescence lifetime. The exact attribution of these regions requires further study, but it should be pointed out that, depending on the cultivation conditions and growth substrate, the mitochondria may form large structures circling the cell boundary ${ }^{40}$, so it would be tempting to interpret the images in this light. From Figure 7c, it is also possible to make estimates of the viscosity making use of the DASPMI lifetime, assuming (and this is a moot point) that the dye's photophysics are not significantly influenced by other factors. This leads to the values on the associated lifetime bar to relate to viscosities from $\sim 40$ to $3000 \mathrm{cP}$. The lower value is roughly twice that obtained by the TRES analysis unsurprising as there should not be any influence from DASPMI in solution. The results presented here are used for demonstrative purposes only, but show the potential use of the fluorescence lifetime to provide an extra parameter by which to improve image contrast and by the fact that it is an absolute measurement provide quantative data in cellular systems.

\section{CONCLUSION}

In this work we demonstrate two biologically relevant application examples based on the use of a time-resolved fluorescence microscope containing a matched high repetition rate semiconductor laser excitation source with low dead time timing electronics. The elucidation of the influence of a conducting metal surface, formed in situ using the microscope's excitation source in CW mode with silver nitrate incorporated within a sol-gel film, on the fluorescence of a FITC tagged protein was performed. An example of cellular imaging was carried out using DASPMI stained yeast. Making use of this probe's photophysical properties and the measurement of fluorescence lifetime enables the potential evaluation of intracellular viscosity to be made.

\section{ACKNOWLEDGEMENT}

The authors wish to acknowledge the invaluable contributions of Scott Finnigan and Shaun Gellie (Glasgow Caledonian University) to the section relating to silver nanostructure preparation and characterisation. Marina Kuimova and Gokhan Yahioglu (Imperial College London and PhotoBiotics London, respectively) are also thanked for the provision and synthesis the bodipy derivative used in this work. 


\section{REFERENCES}

[1] Suhling, K., French, P.M.W. and Phillips, D., "Time-resolved fluorescence microscopy," Photochem. Photobiol. Sci., 4, 13-22 (2005).

[2] Coates, P.B., "The correction for photon "pile-up" in the measurement of radiative lifetimes," J. Phys. E: Sci. Instrum. 1, 878-879 (1968).

[3] Walker, J.G., "Iterative correction for "pile-up" in a single-photon lifetime measurement," Opt. Commun., 201, 271-277 (2002).

[4] Davis, C.C. and King, T.A., "Correction methods for photon pile-up in lifetime determination by single-photon counting,” J. Phys. A: Gen. Phys. 3, 101-109 (1970).

[5] Fleishmann, M., Hendra, P.J. and Mcquilan, A.J., "Raman-spectra of pyridine adsorbed at a silver electrode," Chem. Phys. Lett., 26, 163-166 (1974).

[6] Lakowicz, J.R., "Plasmonics in biology and plasmon-controlled fluorescence," Plasmonics, 1, 5-33 (2006).

[7] Geddes, C.D. and Lakowicz, J.R, “Metal enhanced fluorescence," J. Fluorescence, 12, 121-129 (2002).

[8] Fu, Y. and Lakowicz, J.R., "Single-molecule studies of enhanced fluorescence on silver island films," Plasmonics, 2, 1-4 (2007).

[9] Hayakawa, T., Selvan, S.T. and Nogami, M., "Field enhancement effect of small Ag particles on the fluorescence from $\mathrm{Eu}^{2+}$-doped $\mathrm{SiO}_{2}$ glass," Appl. Phys. Lett., 74, 1513-1515 (1999).

[10] Aslan, K., Lakowicz, J.R. and Geddes, C.D., "Metal-enhanced fluorescence using anisotropic silver nanostructures: critical progress to date," Anal. Bioanal. Chem., 382, 926-933 (2005).

[11] Maliwal, B.P., Malicka, J., Gryczynski, I., Gryczynski, Z. and Lakowicz, J.R.," Fluorescence properties of labelled proteins near silver colloid surface," Biopolymers, 70, 585-94 (2003).

[12] Brinker, C.J. and Scherer, G.W., Sol-gel science, Academic Press, New York. (1989).

[13] Hench, L.L. and West, J.K., "The sol-gel process," Chem. Rev., 90, 33-72 (1992).

[14]Hungerford, G., Rei, A., Ferreira, M.I.C., Allison, A. and McLoskey, D., "Application of fluorescence techniques to characterise the preparation of protein containing sol-gel derived hosts for use as catalytic media," Prog. React. Kin. Mech., 34, 289-327 (2009).

[15] Hungerford, G., Rei, A., Ferreira, M.I.C., Suhling, K. and Tregidgo, C., "Diffusion in a sol-gel derived medium with a view towards biosensor applications," J. Phys. Chem. B, 111, 3558-3562 (2007).

[16] Hungerford, G., Rei, A. and Ferreira, M.I.C., "Use of fluorescence to monitor the incorporation of horseradish peroxidase into a sol-gel derived medium," Biophys. Chem., 120, 81-86 (2006).

[17] Livage, J., Coradin, T. and Roux, C., "Encapsulation of biomolecules in silica gels," J. Phys.:Condens. Matter, 13, R673-R691 (2001).

[18] Kawashita, M., Tsuneyama, S., Miyaji, F., Kokubo, T., Kozuka, H. and Yamamoto, K., “Antibacterial silvercontaining silica glass prepared by sol-gel method," Biomaterials, 21, 393-398 (2000).

[19] Jeon, H.-J., Yi, S.-C. and Oh, S.-G., "Preparation and antibacterial effects of $\mathrm{Ag}_{-} \mathrm{SiO}_{2}$ thin films by sol-gel method," Biomaterials, 24, 4921-4928 (2003).

[20] Babapour, A., Akhavan, O., Azimirad, R. and Moshfegh, A.Z., "Physical characteristics of heat-treated nanosilvers dispersed in sol-gel silica matrix," Nanotechnol., 17, 763-771 (2006).

[21] Rentería, V.M. and García-Macedo, J., "Modeling of optical absorption of silver prolate nanoparticles embedded in sol-gel glasses," Mat. Chem. Phys., 91, 88-93 (2005).

[22] Ahmad, M.B., Shameli, K., Darroudi, M., Yunus, W.M.Z.W. and Ibrahim, N.A., Am. J. Appl. Sci., 6, 20302035 (2009).

[23] De Cremer, G., Antoku, Y., Roeffaers, M.B.J., Sliwa, M., Van Noyen, J., Smout, S., Hofkens J., De Vos, D.E., Sels, B.F. and Vosch, T., "Photoactivation of silver-exchanged zeolite A," Angew. Chem. Int. Ed., 47, 28132816 (2008).

[24] Hungerford, G. and Ferreira, J.A., "The effect of the nature of retained solvent on the fluorescence of Nile Red incorporated in sol-gel-derived matrices," J. Luminescence, 93, 155-165 (2001).

[25] Hungerford, G., Pereira, M.R., Ferreira, J.A., Viseu, T.M.R., Coelho, A.F., Ferreira, M.I.C. and Suhling, K., "Probing Si and Ti sol-gel matrices by fluorescence techniques," J. Fluorescence, 12, 397-417 (2002).

[26] Wang, W. and Asher, S.A., "Photochemical incorporation of silver quantum dots in monodisperse silica colloids for photonic crystal applications," J. Am. Chem. Soc., 123, 12528-12535 (2001). 
[27] Hungerford, G., Toury, M., McLoskey, D., Finnigan, S., Gellie, S. and Holmes-Smith, A.S., "In situ formation of silver nanostructures produced via laser irradiation within sol-gel derived films and their interaction with a fluorescence tagged protein," Phys. Chem. Chem. Phys., 12, 4696-4701 (2010).

[28] Bereiter-Hahn, J., "DASPMI as a fluorescent probe for mitrochondria in situ," Biochim. Biophys. Acta, 423, 114 (1976).

[29] Strehmel, B. and Rettig, W., "Photophysical properties of fluorescence probes I: dialkylamino stilazolium dyes," J. Biomed. Optics, 1, 98-109 (1996).

[30] Strehmel, B., Seifert, H. and Rettig, W., "Photophysical properties of fluorescence probes. 2. A model of multiple fluorescence for stilbazolium dyes studied by global analysis and quantum chemical calculations," J. Phys. Chem. B, 101, 2232-2243 (1997).

[31]Rei, A., Hungerford, G., Ferreira, M.I.C., "Probing local effects in silica sol-gel media by fluorescence spectroscopy of p-DASPMI," J. Phys. Chem. B, 112, 8832-8839 (2008).

[32] Hungerford, G., Allison, A., McLoskey, D., Kuimova, M.K., Yahioglu, G. and Suhling, K., "Monitoring sol to gel transitions via fluorescence lifetime determination using viscosity sensitive fluorescent probes," J. Phys. Chem. B. 113, 12067-12074 (2009).

[33] Kemnitz, K., New Trends in Fluorescence Spectroscopy, Springer-Verlag, Berlin Heidelberg, 381-410 (2001).

[34] Förster, T. and Hoffmann, G., "Viscosity dependence of fluorescent quantum yields of some dye systems," Z. Phys. Chem., 75, 63-76 (1971).

[35] Wilhelmi, B., "Influence of solvent viscosity on the excited-state lifetime and fluorescence quantum yield of dye molecules," Chem. Phys., 66, 351-355 (1982).

[36] Boens, N., Qin, W., Basarić, N., Hofkens, J., Ameloot, M., Pouget, J., Lefèvrer, J.-P., Valeur, B., Gratton, E., vande Ven, M., Silva Jr, N.D., Engelborghs, Y., Willaert, K., Sillen, A., Rumbles, G., Phillips, D., Visser, A.J.W.G., van Hoek, A., Lakowicz, J.R., Malak, H., Gryczynski, I., Szabo, A.G., Krajcarski, D.T., Tamai, N. and Miura, A., "Fluorescence lifetime standards for time and frequency domain fluorescence spectroscopy," Anal. Chem., 79, 2137-2149 (2007).

[37] Kuimova, M.K., Yahioglu, G., Levitt, J.A. and Suhling, K., "Molecular rotor measures the viscosity of live cells via fluorescence lifetime imaging," J. Am Chem. Soc., 130, 6672-6673 (2008).

[38]Köllner, M. and Wolfrum, J., "How many photons are necessary for fluorescence lifetime measurements," Chem. Phys. Lett., 200, 199-204 (1992).

[39] Hungerford, G., Suhling, K. and Ferreira, J.A., "Comparison of the fluorescence behaviour of rhodamine 6G in bulk and film tetraethylorthosilicate derived sol-gel matrices," J. Photochem. Photobiol. A: Chem., 129, 71-80 (1999).

[40] Visser, W., van Spronsen, E.A., Nanninga, N., Pronk, J.T., Kuenen, J.G. and van Dijken, J.P., "Effects of growth conditions on mitrochrondrial morphology in Saccharomyces cerevisiae," Ant. van Leeuwenhoek, 67, 243-253 (1995).

[41] Herskowitz, I., "Lifecycle of the budding yeast Saccharomyces cerevisiae," Microbiol. Rev., 52, 536-553 (1988).

[42] Weisman, L.S., Bacallao, R., Wickner, W., "Multiple methods of visualizing the yeast vacuole permit evaluation of its morphology and inheritance during the cell cycle," J. Cell Biol., 105, 1539-1547 (1987).

[43] Davenport, L., Knutson, J.R., Brand, L., "Excited state proton transfer of equilenin and dihydroequilenin interaction with bilayer vesicles," Biochem., 25, 1186-1195 (1986).

[44] Burns V.W, "Microviscosity and calcium exchange in yeast cells and effects of phenethyl alcohol," Exp. Cell Res., 64, 35-40 (1971).

[45] Puchkov, E.O., "Brownian motion of polyphosphate complexes in yeast vacuoles: characterization by fluorescence microscopy with image analysis," Yeast, 27, 309-315 (2010).

[46] Fisher, J.K., Ballenger, M., O’Brien, E.T., Haase, J., Superfine, R. and Bloom, K., "DNA relaxation dynamics as a probe for the intracellular environment," Proc. Natl. Acad. Sci., 106, 9250-9255 (2009). 